\title{
Génesis y tensiones en la comunidad societal de Parsons
}

\section{Genesis and tensions on Parsons' Societal Community}

doi: http://dx.doi.org/10.32870/

espiral.v25i71.6574

Francisco Cantamutto*

\section{Resumen}

El presente artículo explica la génesis y el desarrollo del concepto de comunidad societal del sociólogo funcionalista Talcott Parsons. Se parte del supuesto de que dicho concepto implicó cierta novedad respecto a los planteamientos iniciales de Parsons. El artículo ofrece un rastreo del concepto siguiendo la obra del propio Parsons y la bibliografía especializada, que se procura sintetizar.Se presentan al final dos tensiones del propio concepto: respecto de la inclusión de grupos no miembros y el uso de la coerción.

Palabras clave: comunidad societal, orden social, Parsons, génesis y desarrollo, funcionalismo.

\begin{abstract}
This article explains the genesis and development of the concept of societal community, created by functionalist sociologist Talcott Parsons. It is assumed that societal community concept involves certain novelty. This article shows the paths of this concept following the works of Parsons as well as specialized bibliography, in an attempt to synthesize it. Finally, the article presents two internal tensions of the concept itself: regarding the inclusion of non-member groups and the use of coercion.
\end{abstract}

Keywords: Societal community, social order, Parsons, genesis and development, functionalism.

\footnotetext{
- Profesor-Investigador en el Instituto de Altos Estudios Sociales, Universidad Nacional de San Martín-CONICET,Argentina. ORCID: http://orcid.org/0000-0002-6996-1534.

franciscojcantamutto@gmail.com

Fecha de recepción: 27 de febrero de 2017. Fecha de aceptación: 14 de agosto de 2017.
} 


\section{Introducción}

Los sociólogos clásicos de la segunda generación escribieron entre finales del siglo XIX y principios del XX, cuando los cambios propulsados por las revoluciones industrial y democrática habían comenzado a afianzarse (Alexander, 1990). La idea elemento (Nisbet, 1996) de comunidad sirvió para la formulación de un par dicotómico, fructífero en términos del desarrollo de la disciplina sociológica, con la idea de sociedad. Tönnies ofrecería la primera proposición en estos términos y el par dicotómico sería retomado a partir de allí en diferentes autores y obras (Sasín, 2012, p. 73).

De Marinis (2010b) propone la posibilidad de aprehender esta dicotomía a través de tres claves complementarias de lectura. En primer lugar, pensando que esta propuesta está asentada en la intención de dar cuenta del proceso de los cambios detectados por los autores clásicos como signo de su época (tipo histórico); la instalación del doble par permitía describir un cambio en una dirección específica (quizá porque los autores estaban aún imbuidos de las nociones lineales de tiempo tan típicas por entonces), que iba de la comunidad a la sociedad. En segundo lugar, pensando en la intención detrás de ella de la identificación de características prototípicas de las relaciones entre los hombres para la descripción "aséptica" de la realidad en función de la presencia o no de ciertos parámetros bien definidos (el tipo ideal). Finalmente, incluso cuando hubiera pretensiones de neutralidad valorativa, la dicotomía hacía las veces de fuente de crítica a la realidad presente, definiendo aquello que ya no está o aquello que falta, y, por ello mismo, sirviendo de base para la posibilidad de buscar ciertos cambios (el tipo utópico).

El mismo autor propone visualizar la (re)aparición del concepto comunidad en la etapa ligada a los años del Estado de bienestar, o welfarismo (De Marinis, 2005). El teórico por 
excelencia de la sociología de esta etapa es, para bien o mal, Parsons. La tematización de la comunidad en Parsons llega relativamente tarde: es en las obras de sus últimas etapas que aparece explícitamente el concepto de comunidad societal (en adelante, CS). De hecho, será por primera vez en el libro La sociedad: perspectivas evolutivas y comparadas (de 1966) donde se enuncie este concepto, propuesto ahí en un sentido muy diferente al de los autores clásicos.

Según afirma el propio Parsons (1986, p. 55), la dicotomía comunidad-sociedad no permite una buena lectura de la realidad, principalmente, porque contiene las reminiscencias idealizantes de un pasado que cambió. Sin la nostalgia por las relaciones cálidas adjudicadas a la comunidad ni el pesimismo por el desgajamiento de la sociedad, Parsons celebró el (supuesto) pluralismo de su tiempo con un optimismo endémico (Holton y Turner, 1986). Pero, lo que es más, con su propuesta conceptual de la CS, Parsons logra sintetizar - paradójicamente- ambos polos de la dicotomía (Sasín, 2012; Sciortino, 2005).

Parsons se encontraba fascinado con la capacidad de la sociedad contemporánea para lidiar con la tensión emergente de la desigualdad producida por el mercado y la igualdad propuesta por la democracia (Boasso, 1980; Holton y Turner, 1986), en un optimismo que rayaba lo celebratorio o apologético (Alexander, 2005). De Marinis (2010b) señala que, de las tres claves de lectura antes señaladas, con Parsons la que aplica con más justeza es la de tipo utópico, esto es, como conciencia de la racionalidad keynesiana.

No sería cierto afirmar que el concepto irrumpe desde el vacío en la obra del autor. Como muestran Fox, Lidz y Bershady (2005), se trata de una lenta maduración dentro de las líneas fundamentales de su pensamiento (Sadrinas, 2012). Sciortino (2007), por su parte, señala su entrelazamiento con otras preocupaciones de Parsons en esa época: los medios generalizados de intercambio y la teoría de la 
evolución. De Marinis (2010a) señala que existen al menos dos líneas de continuidad en la obra de Parsons, ligadas a sendos problemas que lo aquejaron permanentemente: el problema del orden -y su solución hobbesiana- y el de los componentes no racionales de la acción.

La tarea propuesta para este artículo es analizar la génesis y las tensiones del concepto CS de Parsons. Esto es relevante por diversos motivos. En primer lugar, cada nuevo recorrido ofrece luces sobre una de las obras de la sociología más influyentes del siglo XX. En este sentido, aquí apenas buscamos ofrecer una síntesis sobre parte de la abundante bibliografía existente del tema, con un explícito fin propedéutico. En segundo lugar, estas miradas se realizan siempre desde el presente, en el cual tanto desde el ángulo de la teoría social como desde los debates públicos aparecen intensas polémicas sobre la constitución de las sociedades en tiempos de la mundialización.

La nación como eje de la inclusión y el rol de allí derivado para el Estado han sido puestos en cuestión por vías diversas en las últimas décadas: la liberalización asimétrica de los movimientos de capitales y de personas, con sus efectos diferenciales en términos de derechos, la creciente complejización de orientaciones de valor -entre las que se destaca el impacto cultural incorporado en los nuevos patrones de consumo y el acceso a nuevas tecnologías-, o la creciente pauperización global de las condiciones de vida de la clase trabajadora -que pone dudas sobre la inclusión política de una parte significativa de las sociedades- son apenas algunos ejemplos de esto. Las dificultades que tienen las sociedades para lidiar con estos cambios, en especial los impulsados por la creciente integración económica desprovista de un cambio político equivalente, han mostrado un reciente resurgimiento de tensiones políticas locales y globales, asociadas a fundamentalismos y la reclusión en las comunidades locales. Por supuesto, esta historia no está 
sellada, y las reflexiones que aquí se ofrecen pueden servir de herramientas para pensar estas tensiones.

El presente artículo parte de los planteamientos iniciales del autor y llega hasta su esquema evolucionista. El artículo se apoya en la abundante bibliografía existente, buscando ofrecer una síntesis pedagógica de la misma. Se plantean al final dos tensiones internas al concepto, que, según entendemos, ponen cota a los alcances democráticos que Parsons le atribuye a su propuesta. El interés expreso de Parsons por la integración social le dificultaría incorporar ciertas tensiones constitutivas de las sociedades contemporáneas.

\section{El problema del orden y la solución utilitarista}

En sus primeras obras, Parsons (1968) discutió abiertamente con los planteamientos utilitaristas para explicar la sociedad. Estos retoman el sensualismo de la Antigüedad, que asume que todo acontecer existencial es evaluado en términos de placer o displacer provocado al individuo. En su versión moderna, esta corriente propone como guía de la acción a la maximización de la utilidad individual, proponiendo al individuo como mejor juez para tal determinación.

Según Parsons (1967a; 1968), cuatro serían las características de este enfoque: 1) atomismo: asume como unidad de análisis al individuo, estando este desposeído de capacidad de imponerse al resto de modo unívoco; 2) racionalismo: los individuos tienen plena racionalidad en la determinación de los elementos que componen la situación donde se maximiza el alcance de fines; 3) carácter fortuito de los fines: no hay dentro de la teoría ninguna restricción que permita predecir los fines que los individuos perseguirían, ya que estos son determinados exógenamente y, por tanto, aleatorios para el sistema; 4) empirismo: toma por método la acumulación de casos o experiencias, proyectando a la totalidad social características halladas (y, por ello, sólo válidas) en las partes. 
Dado que el único criterio para aceptar una regla general es -para el utilitarismo- que esta aporte en todos los casos a la maximización de la utilidad personal, lo cual es altamente improbable, no podríamos prever la aceptación de ninguna regla de aplicación generalizada, universal (Münch, 1982). El enfoque utilitarista sería entonces incapaz de explicar el orden social, es decir, la existencia de cierta estabilidad empírica en la organización (cualquiera que sea esta) del mundo social. Y, sin embargo, la sociedad no se desintegra: ¿cómo se explica esto? ${ }^{1}$

Esta situación hobbesiana sólo sería solucionada a partir de la imposición de un orden por vía coercitiva (Alexander, 1989). Esta sería, sin embargo, una falsa solución, que traslada el problema a la disputa por el control del órgano con capacidad de imposición coercitiva (Münch, 1982). Por lo demás, no altera sustancialmente el problema: la aplicación de sanciones sólo agrega un elemento más en la ecuación de costos y beneficios del cálculo individual. La estabilidad no se alcanzaría por esa vía, lo que constituye el dilema (aporía) utilitarista: la necesidad de oscilar entre una salida liberal (postular pequeñas unidades interactuantes con capacidad mutua de control) y una salida absolutista (que una unidad tenga capacidad unívoca de imponerse al resto).

Una salida a este dilema sería la construcción de un nosotros (Münch, 1983): un marco de referencia colectivo, formado a partir de la interacción discursiva y la construcción de consenso. Este fue el camino de la primera teoría (voluntarista) de la acción de Parsons (1968): constituir una tradición normativa común compone un límite a la acción, al constreñir el campo de elegibles.

I. Sciortino (2007) señala que esta preocupación de la integración social es el centro de la reflexión parsoniana, algo que la teoría social posterior habría descuidado. Esta idea es llamativa, puesto que, por ejemplo, los esfuerzos de Habermas (1998) y de Luhman (1998) están claramente centrados en esta explicación a través de la comunicación. 
Parsons intentó así combinar el racionalismo utilitarista con la importancia de la normatividad en la acción (tesis de la convergencia). Para esto, rastreó en Weber, Durkheim, Marshall y Pareto los elementos que le permitían explicar la emergencia de la teoría voluntarista. ${ }^{2}$ Según esta, las normas no resultan una exterioridad al individuo actuante, sino que forman parte constitutiva de la definición misma de la situación y los fines que el actor persigue. La norma, internalizada a través de la socialización, limitaría, a la vez que permite, los cursos de acción. De este modo, el conjunto de los fines asequibles y los medios utilizables se restringen, desde el propio actor. A partir de la existencia de patrones de valor compartidos, los individuos incorporan ciertos cursos de acción como motivaciones particulares, lo que permite establecer el orden como una premisa explicada, en lugar de una conclusión derivada. El orden social estaría basado idealmente en la integración en un sistema de valores comunes (Parsons, 2007, p. 69; Sasín, 2012, p. 81).

La distancia respecto de un normativismo a secas estaría en el elemento voluntario del actor. Así como la propuesta kantiana entiende que las reglas morales requieren para su aplicación no sólo de razón -distinguir entre el bien y el mal-, sino también voluntad -de seguir el camino que la razón distingue-, Parsons entendía que la norma propone valores que motivan, pero que el actor siga o no este camino es un problema volitivo, no una imposición externa. Los sujetos, señaló Parsons (1968, p. 118), no responden sólo a estímulos, sino que tratan de ajustar su acción a modelos deseados.

2. La exclusión de Marx entre sus fuentes de inspiración no es casual, pues Parsons era reticente a explicar la constitución del orden social a partir de clases sociales, y en particular a considerar el conflicto social como eje de la misma. El liberalismo económico clásico ya abordaba lo primero como básico, y Hegel enfatizó el rol del Estado al incorporar el segundo punto. Marx relacionó ambos elementos con el modo de producción, completando el recorrido. 
Esta normatividad afecta al conjunto de los elementos del acto-unidad: a la definición de la situación (condiciones y medios) tanto como a los fines perseguidos. La existencia misma de elementos normativos explica el orden en la sociedad. La pregunta planteada desde el principio es, entonces, de dónde emergen los modelos deseados. Evidentemente, su origen no puede ser el individuo: es ineludible una referencia a los demás (los otros). Esto llevó a Parsons a problematizar y generalizar su esquema original.

\section{Del acto-unidad al problema de la integración}

Luego de la Estructura de la acción social (de 1937), Parsons publicó varios trabajos, muchos de ellos en referencia a problemas políticos de su época. En ellos, se perfila con claridad un razonamiento que ampliaría luego: el rol de la socialización para la integración entre normas y pautas de comportamiento. En varios de los artículos compilados como Ensayos de teoría sociológica (1967a), de 1947, propone a la educación y la propaganda como las principales formas de afectar los sentimientos y las pautas de referencia de los sujetos. En su siguiente gran obra, El sistema social, de 1951, se produce un cambio de atención: el proceso de interacción constituye un esquema relacional permanente, constituyendo un sistema. Fue un paso importante en el giro -nunca completo- desde una teoría de la acción a la reflexión sobre la teoría de sistemas (Luhman, 1995; Parsons, 1977, p. 234; Almaraz, 1979).

La unidad básica del acto-unidad pasa a ser analizada bajo la organización de un sistema: los actores interactúan con base en ciertas estructuras de relación, según se encuentran ocupando posiciones (status) que suponen ciertos procesos (rol), como atributos del propio sistema (Parsons, 1966, pp. 33-34). La orientación motivacional de los actores (búsqueda de gratificación o de evitar la privación) depende de 
ciertas pautas, que, por su relativa estabilidad, no parecen depender de la situación. La estabilidad de ciertos símbolos y valores remite a una característica del sistema y no de la acción contingente del actor. De hecho, el actor, imbuido en interacciones cotidianas con otros actores, comienza a esperar que esas pautas se validen en la acción de los otros, logrando de esa forma adecuar sus expectativas.

Parsons (1966, p. 16) propuso las cuatro dimensiones del sistema general de la interacción humana: la personal, la social, la cultural y la biológica. ${ }^{3}$ La acción humana, marco de referencia común, se compone de elementos de los cuatro subsistemas. El orden se produce por la integración de las motivaciones de los actores con criterios normativos culturales, y la estabilidad de la interacción es producto de criterios comunes de valor (1966, pp. 25 y 49). La integración en el sistema total de la acción es un compromiso -nunca perfecto- entre las tensiones de consistencia de sus componentes sociales, culturales y de personalidad (1966, pp. $26-27){ }^{4}$

En otros términos, el sistema social requiere que los actores incorporen pautas valorativas comunes a sus personalidades, que guíen la acción en ciertos sentidos y no en otros. Para motivarlos correctamente, el sistema recurre a la socialización: lograr que el actor incorpore a la personalidad pautas normativas necesarias para la subsistencia del sistema. Es indistinto si los actores incorporan las normas por conveniencia o por introyección: el sistema deberá encontrar gratificaciones y compensaciones para

3. Esta última ya aparecía, aunque menos desarrollada, en el planteamiento de la Estructura de la acción social (1968, p. 85).

4. Sciortino (2007) señala que esta tensión irresuelta es sustancial al funcionamiento de la sociedad, y no una categoría residual, con lo que la acusación a Parsons respecto de su fascinación por el orden social, desatendiendo los cambios, parecería algo exagerada. No obstante, un lector atento de la obra de Parsons ha señalado que, a pesar de reconocer las tensiones, estas aparecen como contingentes ante la necesidad funcional de estabilidad (Luhman, 1995, p. 44). 
las expectativas generadas en los actores, cuya realidad no necesariamente coincidirá con el ideal proyectado. El sistema necesita, además, cubrir las necesidades básicas de los actores. En este sentido, debe resolver la asignación de recursos de modo que maximice las capacidades actuales y futuras. La integración y la asignación de recursos serán los principales problemas del sistema social como tal (Fox, Lidz y Bershady, 2005).

Según se enuncia en El sistema social (1966, pp. 36-40), los prerrequisitos funcionales de los sistemas sociales son: 1) cumplir las condiciones de funcionamiento orgánico; 2) generar las motivaciones de rol y evitar la conducta lesiva; y 3) lograr el mantenimiento de pautas culturales que garanticen un mínimo de orden. Son funciones que entablan relaciones directas entre los distintos subsistemas. Parsons hace explícita la doble dimensión involucrada: la socialización (para internalizar las pautas) y el control social (para quien no cumple lo anterior). Es decir, la propuesta de un orden no basado en la coerción para solucionar el problema utilitario choca contra las necesidades funcionales del sistema.

Más allá de esta ambivalencia, la propuesta de Parsons es clara. El sistema social requiere para su sostenimiento de lograr cierta integración, cierto compromiso entre los valores del actor y los del sistema. Los prerrequisitos funcionales adoptarán en las siguientes obras de Parsons una autonomía propia, al asociarse a las funciones de los diferentes subsistemas (Mitchell, 1967, pp. 55-58). Aun cuando no encontramos aquí la noción de comunidad de manera explícita, comenzamos a verificar que las necesidades de estabilidad giran su énfasis hacia la estructura de la interacción, que -por definición-involucra a más de un actor. Es decir, el problema del orden no tiene solución en la escala del actor individual: es necesaria una perspectiva sistémica. 


\section{El análisis tetradimensional}

Parsons desarrolló una generalización empírico-conceptual en el modelo conocido por el acrónimo AGIL (por sus términos en inglés). Münch (1982; 1990) propone entender este esquema como la continuidad de los elementos del actounidad, combinando nuevos ejes, como se ve en la Figura 1. Las iniciales de AGIL refieren a los cuatro problemas o funciones del sistema general de la acción, caracterizado como un sistema abierto (Almaraz, 1979; De Marinis, 2010b; Fox, Lidz y Bershady, 2005; Luhman, 1995):

1. Adaptación: refiere al control generalizado sobre los intercambios con el ambiente, orientado a la satisfacción de las exigencias situacionales. El sistema tiene que adaptarse a su entorno y lograr que este se adapte a él. Requiere la generación de recursos y su asignación entre los actores. El organismo conductual se especializa en el cumplimiento de esta función. En relación a la acción, se encuentra en el máximo de complejidad simbólica (la necesidad de interpretar es mayor) y mayor contingencia, es decir, menor control por parte del sistema: se trata de ajustar los medios -disponibles-para alcanzar ciertos fines - parcialmente generados por el ambiente-, ambos en un marco de amplia variabilidad.

2. Logro de metas: son los procesos de organización de las actividades en la forma de esfuerzos concertados por alcanzar ciertas metas primordiales. Definir esas metas y alcanzarlas es función del sistema de la personalidad. Respecto a la acción, implica menor contingencia (mayor control) y mantiene una elevada complejidad simbólica: se trata del ajuste más regulado de los objetivos a alcanzar, pretendidamente controlados por la socialización de las personas, que, en cualquier caso, tienen que interpretar la situación en la que se encuentran. 
3. Integración: refiere a los procesos de ajuste mutuo entre unidades sociales, su interrelación, para garantizar la lealtad, la adhesión y la interdependencia. Implica el establecimiento de prioridades entre unidades y la institucionalización de un orden normativo común junto a mecanismos de control social. La integración es la contrapartida de la adaptación, en el sentido de organizar el ambiente interno del sistema. La diferenciación de unidades en el sistema requiere que se desarrollen mecanismos que creen solidaridad y eviten los conflictos. Es la tarea que cumple el sistema social. La acción aquí tiene menor contingencia y menor complejidad simbólica: las normas están allí para ser cumplidas, y existe toda una tradición y costumbre detrás que indica cómo comportarse, sin necesidad de grandes interpretaciones.

4. Mantenimiento de pautas (latencia): se trata de asegurar la adhesión a largo plazo según ciertos valores compartidos que sirven de marco de referencia distintivo para la acción: las pautas de comportamiento comunes. La socialización y la aculturación (en sentido amplio) son los principales procesos, buscando garantizar una base de identidad para el sistema. Se encarga de ello el sistema cultural. La acción aquí requiere de poca interpretación (baja complejidad simbólica), pero alta contingencia, puesto que compartir una serie de valores no restringe tanto el campo de cursos de acción a seguir como para hacerlos previsibles -de ahí la importancia que se plasmen en un orden normativo específico-. 
Figura 1. Esquema AGIL

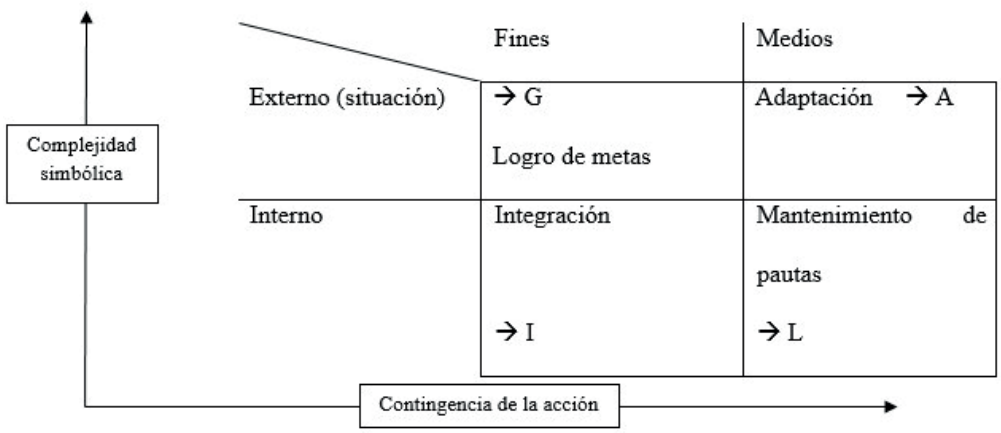

Fuente: elaboración propia con base en Almaraz (1979) y Münch (1980).

La función de integración implica la creación y sostenimiento de una solidaridad básica entre los actores sociales, en un sentido similar al de Durkheim (Parsons, 1977, p. 250; 2007, p. 56; Sciortino, 2005). ${ }^{5}$ A Parsons lo mueve una preocupación por el orden social: ¿cómo reducir la contingencia ligada a las relaciones con el ambiente y a la interpretación -de aquel y de los valores abstractos compartidos-, de modo que se logre una convergencia estable de la sociedad? El sistema social tiene que traducir los valores en normas concretas, que especifiquen cursos de acción concretos, y de ese modo organizar las relaciones internas (Münch, 1994; Parsons, 2007).

Sistema social no es igual que sociedad, siendo esta un tipo particular de aquel, caracterizado por la autosuficiencia en relación tanto a la organización y los recursos internos como al provecho obtenido de su ambiente para realizar su cultura normativa (De Marinis, 2010b; Parsons, 1974a). En el análisis del sistema social, con énfasis en las teorías

5. Sasín (2012, pp. 91-93) observa que esta estrategia duplica el problema de la integración del sistema social a la CS. 
cibernéticas de los sistemas integrados, es posible aplicar nuevamente el esquema AGIL, como se ve en la Figura 2.

Figura 2. Esquema AGIL para el sistema social

\begin{tabular}{|c|c|}
\hline Economía & Política \\
\hline $\begin{array}{l}\text { Sistema fiduciario } \\
\mathrm{L}\end{array}$ & Comunidad Societal \\
\hline
\end{tabular}

Fuente: elaboración propia con base en Almaraz (1979) y Münch (1980).

Repitiendo la lógica del modelo previo, reconocemos aquí nuevos subsistemas, ligados a funciones del sistema social (Gerhardt, 2001). A la economía le corresponde el desarrollo y asignación de recursos básicos, cuyo medio de intercambio generalizado es el dinero. La tarea de la política es la coordinación de esfuerzos para metas colectivas, cuyo medio de intercambio generalizado es el poder. La transmisión, mantenimiento y desarrollo de valores compartidos es tarea del sistema fiduciario, cuyo medio de intercambio generalizado son los compromisos de valor. Finalmente, aparece aquí la CS, núcleo integrativo del sistema social, estableciendo marcos compartidos de órdenes normativos (leyes, costumbres, tradiciones, etc.) para delimitar el campo de acción y establecer las bases de confianza y solidaridad entre actores.

La integración indicaría un impulso inherente a la solidaridad, un sentimiento de pertenencia conjunta, impulso regulado por normas para reducir la contingencia de la subjetividad. Esta solidaridad rebasa el respeto mutuo y la contribución al bien colectivo, remitiendo a una identi- 
ficación mutua (Parsons, 2007, p. 56). Frente a la fuerza centrífuga de la individualidad, la integración responde como la fuerza centrípeta que, anclada en valores y creencias colectivas, traduce ideas en normas concretas que orientan la acción (Gerhardt, 2002; Parsons, 2007). Su medio de intercambio generalizado es la influencia, que es la capacidad de hacer que otros tomen las decisiones esperadas, buscando su anuencia, sea porque lo asumen como algo bueno o porque temen las sanciones de comportarse de otro modo (Gerhardt, 2001). Así, la eficacia de la influencia expresa la confianza en los lazos solidarios detrás de quien ejerce la influencia: nos dejamos influir porque creemos en quien lo hace (Alexander, 1989). Es la función cibernética de control para lograr la autosuficiencia.

Esta expansión del análisis de los medios generalizados de intercambio a diferentes subsistemas se basa en las analogías a partir del dinero, con un extenso desarrollo sobre el medio del poder de Parsons (1977, pp. 204-214). Luhman utiliza explícitamente esta base para expandir la importancia del poder, como medio simbólico, de restringir las selecciones de la comunicación, criticando la efectividad que Parsons le atribuye a este medio, pues su carácter relacional es el origen de la posibilidad de su deflación (Torres Nafarrete, 2004, pp. 93-103). Habermas (1998, p. 102; 2001, pp. 373-374), por su parte, encontrará excesivo y erróneo este desarrollo del argumento: aunque el dinero y el poder dan cuenta de la integración en los subsistemas económico y político, su intromisión en la esfera del mundo de la vida no parece legítima ni fundada.

\section{La CS en el marco evolucionista}

Con base en lo anterior, Parsons recuperó la importancia de la CS dentro de su estudio sobre el cambio en las 
sociedades. ${ }^{6}$ Así, en La sociedad: perspectivas evolutivas y comparadas, de 1966, retoma el problema hobbesiano del orden, planteado en su primera obra, para introducir su solución a través de la CS (1974a, p. 18). La define así:

el núcleo de una sociedad, como sistema, es el orden normativo, organizado dentro de un patrón, a través del que se organiza colectivamente la vida de una población. Como orden, contiene valores y normas diferenciadas y particularizadas, así como reglas, que requieren referencias culturales para resultar significativas y legítimas. Como colectividad, despliega un concepto organizado de membrecía que establece una distinción entre los individuos que pertenecen o no a ella (Parsons, 1974a, p. 24).

Quedan así presentadas las líneas fundamentales de lo que Parsons entendía por CS: una lealtad básica que mantenga unida la pluralidad (Parsons, 1974b, p. 23). Siguiendo el rastreo que realiza Alexander (2005), la CS, como núcleo del sistema social, se desdobla sucesivamente en:

1. Orden: su relevancia fundamental, en continuidad con anteriores planteamientos, remite a la garantía del orden (estabilidad) de la sociedad. Al modo de la solidaridad durkheimiana, debe establecer los lazos básicos de integración y lealtad entre actores, tanto en su semejanza (mecánica) como en su diferencia (orgánica) (De Marinis, 2010b; Sciortino, 2005). Incluye tanto la promoción de ciertas pautas comunes como la corrección de aquellas desviadas.

2. Valores: la referencia constitutiva del orden son los valores compartidos del sistema social, los cuales guían las pautas básicas de comportamiento. 
- Generales: la vida en sociedades complejas (modernas) no puede guiarse por la aplicación de reglas en exceso específicas, a riesgo de resultar excluyentes. Patrones generales de valoración sirven de principios morales como marco universal de justicia (Gerhardt, 2002). Se incluye bajo este paraguas la referencia a nociones últimas de la realidad (Parsons, 1974a, p. 19). Se trata de una cultura compartida.

- Particulares: al mismo tiempo, los valores deben encontrar expresiones específicas que no contradigan los principios generales, esto entre los distintos grupos sociales. De otro modo, el marco de referencia sería demasiado contingente como para garantizar el orden: se requieren especificaciones particularizadas, según la naturaleza del actor social considerado (su posición y rol, relación con otros actores, etc.). Se trata de aspectos particularizados de la cultura.

3. Reglas: ambos conjuntos de valores deben expresarse en un sistema de normas, formales e informales, que garanticen la menor complejidad simbólica de los mismos. Se trata de incrementar la capacidad del sistema de controlar su organización interna, delimitar el campo de lo asequible y fomentar ciertos comportamientos.

4. Colectividad: no trivialmente, la CS define grados de membrecía, según la organización de un núcleo y la existencia de otros actores (individuales o colectivos) que pertenecen al sistema social sin ser parte plena de la CS. Se definen, a fin de cuentas, grados de ciudadanía. ${ }^{7}$ Parsons asocia en la CS abiertamente a la solidaridad en términos durkheimianos con la idea de ciudadanía de T. H. Marshall (Parsons, 1974b, p. 33; 2007, p. 151).

7. Luhman (1998, pp. 176-|8I) señaló que estos grados de ciudadanía componen una estratificación social compleja basada en esquemas de exclusión no uniformes: la pertenencia se regula por la participación autónoma en los diferentes subsistemas funcionales. 
En términos generales, para Parsons (1974a, pp. 24-36; 2007, pp. 76-77) la CS se compone de normas que depositan valores (especificación de abstracciones), y sobre los que se constituye la referencia fundamental para los subsistemas político y económico (control de la contingencia y la complejidad simbólica), responsables de la satisfacción de necesidades sociales: el logro de metas comunes y la asignación de recursos (control de la tecnología).

Las relaciones con los demás subsistemas quedan de plano realzadas: la legitimidad de un orden se basa en su justificación en un conjunto de valores (subsistema cultural); su persistencia requiere tanto de lograr las correctas motivaciones de los actores a través de los procesos de socialización (subsistema de la personalidad) como de su corrección en caso de desvío y de la garantía del suministro de los requisitos básicos para la vida (Parsons, 1974b, pp. 25-29). Si miramos con atención, estas relaciones son expresiones renovadas (tras quince años) de los prerrequisitos funcionales del sistema social.

La CS es el núcleo estable de la sociedad y, por lo tanto, la llave para la puerta del cambio: sin perjuicio de las modificaciones que ocurran, existe un centro gravitacional que sostiene al conjunto social. Quizá esta idea esté influenciada por la disciplina de origen de Parsons, la economía, con su análisis de equilibrios sucesivos; no se trataría de negar el cambio, sino de analizar las tendencias a la estabilidad (Parsons, 2007, p. 147). La dicotomía clásica ha desaparecido: la comunidad ya no expresa un tipo empírico distinguible de la sociedad, sino que expresa su núcleo estructural (sirve como tipo analítico); la comunidad ya no precede en el tiempo a la sociedad, sino que toda sociedad tiene un núcleo comunitario (lo que le permite aplicarla a toda la historia, en clave de evolución). La comunidad ya no remite a imágenes de relaciones cercanas, afectivas, cara a cara, sino que es el 
complejo analítico que funda la permanencia de la sociedad (De Marinis, 2010b).

En clave evolutiva, las inconsistencias entre valores, normas, creencias e intereses son la fuente del cambio social (Mitchell, 1967). El cambio modernizador implica una creciente diferenciación funcional, donde las unidades o los subsistemas ganan en capacidad de adaptación a (nuevos) problemas más específicos. Procesar ese cambio afecta en distinto grado a los diferentes grupos sociales, alterando la estructura social de conjunto (Parsons, 1967a). Si estos grupos ofrecen resistencia, se puede producir un cambio regresivo. Si la diferenciación logra prevalecer, el sistema como tal es capaz de atender más necesidades, en un sentido de especialización del trabajo. Esto implica unidades más diferenciadas, cuya integración se vuelve desafiante, puesto que ya no alcanza con una identidad básica sostenida en la semejanza.

Así, el proceso de cambio como diferenciación implica (Alexander, 1989; Parsons, 1974b; 2007): 1) instituciones cada vez más especializadas, que no es lo mismo que más autónomas: al contrario, dependen cada vez más unas de otras, y por ello es necesario garantizar su ligazón mutua; 2) un criterio más general de pertenencia a la comunidad, que admita la membrecía con base en competencias mínimas y asigne derechos y obligaciones no particulares; 3) valores que se abstraen cada vez más para permitir el consenso. La CS regula este proceso en la medida en que procesa estas modificaciones como necesidad funcional. La necesidad de superar los clivajes de los grupos diferenciados remite a un proceso de pluralismo institucional junto a una ampliación de la ciudadanía plena (Gerhardt, 2001; Parsons, 1974b). Parsons busca así las bases consensuales tácitas (2007, p. 59) de ese nosotros que le preocupa desde sus obras tempranas. 


\section{Tensiones internas en la CS}

Existen algunas tensiones que Parsons refirió, aunque contemplando el riesgo de no vulnerar su noción de CS como paradigma de la solidaridad (Parsons, 2007, p. 148). Resultan particularmente relevantes dos elementos internos: la existencia de grupos sociales no miembros de la CS y el uso de la coerción. ${ }^{8}$ Llamamos la atención sobre ellos porque entendemos que componen el núcleo del problema del orden en Parsons, y la puerta para una crítica interna de su propuesta. Dado el creciente interés del teórico por explicar ese orden utilizando como modelo la sociedad estadounidense de la postguerra, la cuestión de los valores democráticos que supuestamente encarna esta propuesta conceptual no es trivial. Veamos.

\section{I. Grupos no miembros}

En el esquema simplificado de diferenciación, los nuevos grupos son incorporados por una necesidad funcional del sistema, tendiendo al pluralismo de modo natural (Sciortino, 2005, p. 116). En primer lugar, esto soslaya el papel activo de los grupos para lograr su inclusión (y sus disputas históricas), así como las resistencias activas que esto genera entre grupos ya establecidos, reavivando la crítica formulada por Therborn (1973) respecto a que en el esquema parsoniano parece que nadie efectivamente hace algo. No obstante, sería injusto decir que no es posible incorporar esta dimensión a su esquema: de hecho, Sciortino (2005, pp. 124-125) insiste en este punto, al realzar que Parsons identifica el potencial conflicto entre grupos incluidos y grupos buscando inclusión. El pluralismo, más allá de una necesidad funcional, es el resultado de la acción organizada. 
No resulta demasiado arriesgado entender a distintas luchas de minorías como requerimientos de inclusión, sin embargo, resulta problemático no reconocer las distintas dotaciones de recursos, estrategias y capacidades de los diferentes grupos. Esto significa que no todos los grupos obtendrían el mismo nivel de inclusión ni todos serían incluidos a la misma velocidad-aspectos reconocidos por el propio Parsons (1967b)-. Asimismo, tiene escasa atención el hecho de que el carácter asimétrico en la inclusión de ciertos grupos podría reproducirse -e incluso exacerbarse- en el tiempo, esto gracias a las mismas normas y valores que dan estabilidad a la propia CS. Relativo a otros subsistemas (política y economía), es probable que esto se asocie a una distribución asimétrica (y estable) de poder y dinero, que perfectamente puede perpetuarse o intensificarse, dando lugar a mayores tensiones de integración de otros grupos.

Esto nos lleva al segundo punto, que es la distinción misma entre el núcleo de miembros originales y los nuevos grupos buscando membrecía. Aun cuando la incorporación requiere de la abstracción y generalización de las pautas de valores y su expresión normativa (Parsons, 1974a, p. 42), no puede evitarse registrar que existe un núcleo original de valores a abstraer. Incluso sin suponer que se trate de la imposición de valores particularistas del grupo núcleo original, resulta problemático suponer que se trata de una integración plenamente democrática, tal como lo hace Parsons (2007, pp. 58-59) al afirmar el ideal consensual que guía la CS. Sobre este punto, analizando los regímenes políticos, Lechner (1978) enfatizaba la posibilidad de construir acuerdos comunes entre minorías consistentes que luego fueran sometidas al escrutinio general bajo la presentación de un consenso amplio: los grupos no organizados o los que se organizan más tardíamente se ven compelidos a adoptar los marcos de la acción ya establecidos. 
Alexander (2005) señala al respecto una tensión entre la necesidad de integración y la búsqueda de justicia (criterios universales de acción que eviten la vulneración de otros -Parsons, 1974a, p. 30-). La jerarquía moral de los miembros originales se expresa en la universalización de sus pautas para incluir (potencialmente) a los nuevos miembros. Esto expresa justamente lo que en otras corrientes de pensamiento se conoce como construcción de hegemonía, esto es, la prevalencia de un conjunto particular de valores y creencias sobre el conjunto de la población (Gramsci, 1975; Laclau y Mouffe, 1987). La construcción de consenso requerida por el proceso democrático de legitimación involucra crear un sentido común, una tradición que guíe la acción en ciertos sentidos.

La legitimación, en el marco parsoniano, implica que el grupo entrante tiene que lograr las mismas condiciones de humanidad que los miembros originales, en algún sentido o categoría que puede ser nuevo (Gerhardt, 2001, p. 194). Por eso es que Parsons asoció directamente el proceso de expansión de la ciudadanía plena con la tipología de derechos de Marshall (Cohen y Arato, 2000; Parsons, 1967b; 2007; Münch, 1994). Esto es en parte el resultado de la idealización de Parsons de la etapa de la historia que vivió, el welfarismo estadounidense (ver, por ejemplo: Parsons, 2007, pp. 74-75). Sin embargo, la ampliación de la ciudadanía plena a nuevos grupos puede chocar con los intereses, valores o creencias del núcleo de membrecía original. Incluso más: como hemos visto en las sociedades contemporáneas, puede entrar en conflicto con la pertenencia de ciertos grupos ya incorporados (como ocurre con amplios sectores medios, afectados por las políticas de ajuste social ante la crisis).

De hecho, en los años que Parsons desarrollaba estas ideas, la organización keynesiana del sistema-mundo capitalista comenzaba a resquebrajarse, entrando en una fase de crisis que anularía o retraería la mayor parte de 
los derechos ganados durante esta etapa (Duménil y Lévy, 2007). Sasín (2012) afirma que la aparición misma del concepto de CS "[...] podría parecer estar más motivada en los condicionantes del contexto epocal que en desarrollos intrínsecos o necesidades inherentes a la construcción de la teoría [de Parsons]" (p. 78).

A fines de los sesenta, la ampliación de la ciudadanía chocó en términos materiales (e ideológicos) con las necesidades de reproducción del capital, e indujo a una nueva etapa definida genéricamente como neoliberalismo (Harvey, 2007). El nuevo consenso moral se construyó sobre las bases de ciertos valores identificables con ciertas clases, sus necesidades e intereses. Por lo tanto, no resulta trivial la definición de quiénes componen el núcleo de membrecía original.

\subsection{Uso de la coerción}

La segunda tensión estudiada es el rol de la coerción. Aun cuando Parsons buscó desde el comienzo dar cuenta de una salida para explicar el orden sin necesidad del recurso coercitivo, se encontró aquí con un traspié. Justo después de explicar el criterio de justicia, Parsons dice (1974a, p. 30) que "[...] la prevención última de la acción destructiva [sobre la CS] es mediante el empleo de la fuerza física. [...] El control o la neutralización del uso organizado de la fuerza es una necesidad funcional para el mantenimiento de una comunidad societaria". Es decir, la garantía última del orden, la estabilidad, la integración, es el uso de la fuerza (1974b, pp. 26-27), incluso cuando esta es un medio residual para disuadir, del que no se puede abusar sin hacerle perder eficacia (1967c, p. 272).

El uso de la fuerza es controlado por el subsistema político (Parsons, 1974a, p. 33). Según se nos dijo, este está ordenado en referencia a un conjunto de normas que expresan valores, que -según discutimos en el subtítulo anterior- responden 
primaria, aunque no exclusivamente, a un grupo o conjunto de grupos sociales. Es decir, la fuerza garantiza en última instancia un orden fundado en los valores de algunos. Claro que esa garantía sólo aplica como último recurso, pues su uso sistemático produciría una devaluación de su capacidad de sostener el orden (1967c, p. 296). Parsons señaló, contra el análisis marxista, que suponer el monopolio de un grupo en la posición de membrecía real es una dicotomía excesivamente simplificada (1974a, p. 42), pero no pudo ofrecer razones teóricas por las cuales esta posición monopólica o cuasimonopólica no podría sostenerse: ¿qué evita que el grupo constituyente original no aproveche su posición privilegiada para contener, administrar o dosificar el acceso de otros grupos? ¿Qué evita que utilice el recurso coercitivo para este fin, aunque sólo lo haga en situaciones límite? ${ }^{9}$

Quizás por esto, a lo largo de su obra, Parsons reconoció las limitaciones de la orientación normativa y la necesidad de garantizar ciertos medios de control social (Boasso, 1980; Mitchell, 1967). Esto lo llevó a explicitar, desde el principio hasta el final de su carrera, la importancia de la educación como medio de socialización para inculcar ciertos valores particulares (Parsons, 1967a, pp. 206-238; 1986, p. 57).

Cuando en El sistema social explicitó los prerrequisitos funcionales, también realzó la importancia de la correcta motivación y la corrección de conductas lesivas, pero incluso antes en el tiempo, y en relación al problema hobbesiano, ya Alexander (2005) detectó que la salida propuesta por Parsons en la Estructura de la acción social es no democrática. El problema con la imposición coercitiva externa es su baja eficacia, de allí la importancia de la internalización de

9. A esta cuestión -reiteradamente confundida- refiere la noción marxista de dictadura de clase, y no al régimen político -que puede ser democrático (Balibar, 1977)-. 
un cierto orden normativo. ${ }^{10}$ La falta de reconocimiento de Parsons sobre esta tensión no es una ingenuidad: en caso de afirmarla, habría caído su ilusión democrática y pluralista de la sociedad (estadounidense) moderna. ${ }^{11}$

\section{Conclusión}

Talcott Parsons ofreció una reflexión distinguible en relación a la problemática de la comunidad, permitiendo un pasaje a la comprensión de su actualidad en sociedades complejas. El giro propuesto implicó abandonar la polaridad comunidad-sociedad, e incorporarla de manera sistémica al funcionamiento y estabilidad de las sociedades. Ofreció así una explicación al orden social, pero también -y esto no es menor- un programa de acción: cómo reforzar un orden social dado. Según el autor, la acción autointeresada del individuo se limita por las normas compartidas y su efectividad. Según Münch (1983), esta a su vez depende del grado de inclusión en la comunidad, los lazos afectivos, la claridad y conciencia de las normas y el refuerzo a través de rituales y ceremonias. Estas serían, pues, las áreas sobre las cuales habría que intervenir para desarrollar el orden y contener tensiones en sociedades crecientemente complejas.

La omisión teórica de Parsons puede ubicarse en la negación a los aspectos no plenamente democráticos de su propuesta, o mejor dicho, a los límites de su propuesta de democracia. Se trata de la distinción entre grupos miembros

10. Por ello, Dawe ( 1970) afirma que el planteamiento de Parsons sigue señalando una restricción externa al actor, cuya internalización no hace sino afirmar la exterioridad.

I I. La pluralidad es constituida con base en la exclusión de aquellos elementos que cuestionan los fundamentos normativos de la sociedad. Parece que, aun conociendo y estudiando estos fenómenos, no incorpora orgánicamente la década antirroja de los veinte (con hitos como el asesinato de Sacco y Vanzetti) o el macartismo (Parsons, 2007) a la constitución del pluralismo americano. Sin perjuicio de la inteligencia funcional del sistema, esto es un cuestionamiento directo a sus bases democráticas: la exclusión de la comunidad de todo grupo en tensión. 
y no miembros, y el problema del uso de la coerción. Debemos enfatizar que en ambos casos se trata de una crítica interna: lo que Parsons trató de defender una y otra vez en su obra termina sin solución. La propuesta de CS puede ser útil para pensar no sólo los años de la expansión capitalista de la postguerra, sino la propia actualidad, pero, claro, para ello -tal como señalara Alexander (1989)- es necesario reconocer los aspectos no democráticos, de asimetría de poder político, contenidos en la misma.

En este sentido pueden leerse varios de los problemas sociales contemporáneos. La etapa de la mundialización con su asimétrica liberalización del movimiento de capitales y personas- ha intensificado una distribución dispar del poder, el dinero y la influencia, afectando no sólo la incorporación de nuevos grupos dentro de los sistemas sociales nacionales, sino incluso la propia filiación de los grupos ya incorporados. El neoliberalismo, como ofensiva de clase, ha provocado una mayor pauperización de la vida y la pérdida de mecanismos vinculantes de decisión colectiva.

Esto ha fomentado una creciente reacción contra migrantes, pobres e incluso contra las uniones supranacionales (en especial, en los países centrales), poniendo en todos los casos límites a la integración social. Es posible detectar un creciente uso de la fuerza como herramienta para dirimir conflictos a nivel nacional e internacional, como expresión de estas limitaciones.

Por supuesto, este análisis exige la contemplación de los límites de la democracia basada en capacidades asimétricas de generalizar valores y acceso al uso de la coerción. En este sentido, entendemos, la reflexión funcionalista queda trunca por no poder incorporar plenamente el conflicto entre las clases sociales. $\varepsilon$ 
Alexander, J. (1989). Las teorías sociológicas desde la Segunda Bibliografía Guerra Mundial. Madrid: Gedisa.

(1990). "La centralidad de los clásicos", en A. Giddens, J.Turner, et al. (auts.), Teoría social hoy (pp. 22-48). Madrid:Alianza.

(2005). "Contradictions in the Societal Community: The Promise and Disappointment of Parsons' Concept", en R. Fox, V. Lidz, y H. J. Bershady (eds.), After Parsons: A Theory of Social Action for the Twenty First Century (pp. 93-I I0). Nueva York: Russell Sage Foundation.

Almaraz, J. (1979). "La transición del modelo interactivo al sistémico en Parsons”. Reis, 8, 5-32.

Boasso, C. (1980). El estructuralismo funcional de Parsons. Buenos Aires: Instituto de Ciencias Sociales.

Balibar, É. (1977). Sobre la dictadura del proletariado. México: Siglo XXI.

Cohen, J., y Arato, A. (2000). Sociedad civil y teoría política. México: FCE.

Dawe, A. (1970). “The Two Sociologies”. British Journal of Sociology, 2 I, 207-2I8.

De Marinis, P. (2005). "I6 comentarios sobre la(s) sociología(s) y la(s) comunidad(es)”. Papeles del CEIC, 15, I-39.

(20|0a). "Estado y comunidad, de los clásicos a Parsons (y más allá)". Propuesta Educativa, 33, 6I-70.

- (20l0b)."La comunidad societal en Talcott Parsons: un ejercicio para poner a prueba ciertas hipótesis de lectura acerca de la historia del concepto de comunidad en la teoría sociológica" (ponencia presentada en las VI Jornadas de Sociología de la UNLP). Universidad Nacional de La Plata:Argentina.

Duménil, G., y Lévy, D. (2007). Crisis y salida de la crisis. Orden y desorden neoliberales. México: FCE. 
Bibliografía

Fox, R. C., Lidz,V.,y Bershady, H.J. (eds.) (2005). After Parsons: ATheory of Social Action for the Twenty First Century. Nueva York: Russell Sage Foundation.

Gerhardt, U. (200I). "Parsons's analysis of the societal community”, en A. Treviño (ed.), Talcott Parsons Today. His Theory and Legacy in Contemporary Sociology (pp. I77222). Lanham: Rowman \& Littlefield Publishers.

- (2002). Talcott Parsons. An intelectual biography. Cambridge: Cambridge University Press.

Gramsci, A. (1975). Notas sobre Maquiavelo, sobre la política y sobre el Estado moderno. México: Juan Pablos Editor.

Habermas, J. (1998). Facticidad y validez. Madrid:Trotta. (200I). Teoría de la acción comunicativa. Vol. 2. Madrid: Taurus.

Harvey, D. (2007). Breve historia del neoliberalismo. Madrid: Akal.

Holton, R., y Turner, B. ( 1986). Talcott Parsons on Economy and Society. Londres-NuevaYork: Routeledge.

Laclau, E., y Mouffe, C. (1987). Hegemonía y estrategia socialista. Hacia una radicalización de la democracia. Madrid: Siglo XXI.

Lechner, N. (1978). "Poder y orden. La estrategia de la minoría consistente”. Revista Mexicana de Sociología, 40(4), I $20 \mathrm{I}-1258$.

Luhmann, N. (1995). Introducción a la teoría de sistemas. México: Universidad lberoamericana.

(1998). Complejidad y modernidad: de la unidad a la diferencia. Madrid:Trotta.

Mitchell,W. (1967). Sociological analysis and politics. The theories of Talcott Parsons. Nueva Jersey: Prentice-Hall.

Münch, R. (1982).“Talcott Parsons y la teoría de la acción. La constitución del núcleo kantiano”. Revista Internacional de Sociología, 4I, 5 I-85. 
(I983)."From pure methodological individualism to Bibliografía poor sociological utilitarism:A critique o fan avoidable alliance". The Canadian Journal of Sociology, I, 45-77.

(1990). "Teoría parsoniana actual: en busca de una nueva síntesis”, en A. Giddens, J.Turner, et al. (auts.), Teoría social hoy (pp. 155-204). España:Alianza.

(1994).“'La interpenetración de la microinteracción y las macroestructuras en un orden institucional complejo y contingente", en J. Alexander, B. Giesen, R. Münch, y N. Smelser (comps.), El vínculo micro-macro (pp. 385-406). Guadalajara: Gamma.

Nisbet, R. (1996). La formación del pensamiento sociológico I. Buenos Aires:Amorrortu.

Parsons, T. (1966). El sistema social. Madrid: Revista de Occidente.

(I967a). Ensayos de teoría sociológica. Buenos Aires: Paidós.

(1967b)."Full citizenship for the Negro American?", en T.Parsons (aut.), SociologicalTheory and Modern Society (pp. 422-465). Nueva York:The Free Press.

(1967c). "Some reflections on the place of force in social process", en T. Parsons (aut.), Sociological Theory and Modern Society (pp. 264-296). Nueva York:The Free Press.

(1968). Estructura de la acción social. Madrid: Guadarrama.

(1974a). La sociedad: perspectivas evolutivas y comparadas. México:Trillas.

(1974b). El sistema de las sociedades modernas. México:Trillas.

(1977). Social systems and the evolution of action theory. Nueva York:The Free Press.

(1986). Biografia intelectual. El simbolismo económico y religioso en Occidente. Puebla: Universidad Autónoma de Puebla. 
Bibliografía
(2007). American Society. A theory of the societal community. Londres: Paradigm.

Sadrinas, D. (20/2). "La comunidad societal en la obra de Talcott Parsons: tensiones entre la inclusión y la exclusion”, en P. de Marinis (coord.), Comunidad: estudios de teoría sociológica (pp. 265-305). Buenos Aires: Prometeo. Sasín, M. (20I2). “Por qué le habrá puesto 'comunidad”? Una aproximación al lugar de la comunidad societal en la teoría de Talcott Parsons”. Sociológica, 77, 7| - 08.

Sciortino, G. (2005). "How Different Can We Be? Talcott Parsons, the Societal Community and the Multicultural Debate", en R. C. Fox,V. Lidz,y H.J. Bershady (eds.), After Parsons: A Theory of Social Action for the Twenty First Century (pp. I I I- I36). Nueva York: Russell Sage Foundation. (2007). "Introduction: The Action of Social Structure", en G. Sciortino (ed.), American Society. A theory of the societal community (pp. I-53). Londres: Paradigm. Therborn, G. (1973). "Social practice, social action, social magic”. Acta sociológica, 3, I57-I74.

Torres Nafarrete, J. (2004). Luhmann: La política como sistema. México: FCE. 